\title{
Propuesta de una red de censo de mirlo acuático Cinclus cinclus L., 1758 en el País Vasco para detectar tendencias a largo plazo en su distribución a partir de modelos de ocupación.
}

\section{A proposal to survey white-throated dippers Cinclus cinclus L., 1758 in the Basque Country to detect long-term distribution changes with occupancy models.}

José María Sánchez ${ }^{1 *}$, Juan Arizaga ${ }^{1}$, Frank d'Amico ${ }^{2}$

\section{Resumen}

El desarrollo de programas de seguimiento a largo plazo es fundamental para determinar el estado de conservación de poblaciones y especies. El mirlo acuático Cinclus cinclus L., 1758 está incluido en varios listados de especies amenazadas y es, en consecuencia, una especie que tiene interés desde el punto de vista de la conservación y gestión de nuestro patrimonio natural. En la Comunidad Autónoma Vasca (CAV) está catalogado de Interés Especial; está incluido, además, en el Catálogo Español de Especies Amenazadas, así como en el Anexo II del Convenio de Berna. El objetivo de este artículo es proponer un método de censo de mirlo acuático para toda la CAV, basado en modelos de ocupación. Esto se hace con el fin de dotar al territorio de un método útil para determinar, a largo plazo, cambios en la distribución (e, indirectamente, abundancia) de la población. A partir de estudios

\footnotetext{
1 Departamento de Ornitología. Sociedad de Ciencias Aranzadi. Zorroagagaina 11, 20004 Donostia-San Sebastián (Spain).

2 CNRS/Univ. Pau \& Pays Adour, Laboratory of Mathematics and their Applications of Pau. UMR 5142, 64600 Anglet, France. 
previos en los que se calculó el número óptimo de puntos de muestreo para Gipuzkoa dados un valor de $\psi$ (probabilidad de ocupación) y p (probabilidad de detectar la especie si está presente), se obtuvo una red de 199 puntos de muestreo para toda la CAV (zona mediterránea de Álava excluida), que además estaba balanceada desde un punto de vista espacial (ponderada para la longitud de los ríos de cada provincia). La aplicación de este método permitiría censar la especie de estudio a largo plazo, de manera estandarizada, y obtener un valor indicador sobre su estado de conservación. Además, el método de censo sería aplicable, también, para otras especies riparias tales como el martín pescador Alcedo atthis L., 1758 y la lavandera cascadeña Motacilla cinérea L., 1758.

Palabras clave: Aves riparias, paseriformes, programas de seguimiento, modelos de ocupación.

\begin{abstract}
The development of long-term monitoring programmes is essential to determine the conservation status of populations and species. The white-throated dipper, Cinclus cinclus L., 1758 is included in several lists of threatened species and is, consequently, an interesting species from the point of view of conservation and management of our natural heritage. In the Basque Autonomous Community (CAV) it is classified as of Special Interest; it is also included in the Spanish Catalogue of Endangered Species, as well as in Annex II of the Berne Convention. The objective of this article is to propose a census method for the whitethroated dipper, applicable to all the CAV, based on occupancy models. The objective is to provide this Community with a useful method to determine, in the long term, changes in the distribution (and, indirectly, abundance) of the population. From previous studies in which the optimal number of sampling points for Gipuzkoa was calculated given a value of $\psi$ (occupation probability) and $\mathrm{p}$ (detection probability of the species if present), a network of 199 sampling points was obtained for the whole CAV (excluding the Mediterranean zone of Álava), which was also balanced from a spatial point of view (weighted for the length of the rivers of each province). The implementation of this method would allow to survey the studied species and obtain an indicator on its conservation status. Our method could be also used to survey other riparian birds, such as the common kingfishers Alcedo atthis L., 1758 and the grey wagtail Motacilla cinérea L., 1758.
\end{abstract}

Key words: Riparian birds, passerines, tracking programmes, site-occupancy models.

\title{
Laburpena
}

Epe luzeko jarraipen-programak garatzea funtsezkoa da populazioen eta espezieen kontserbazio-egoera zehazteko. Cinclus cinclus L., 1758 ur-zozoa espezie mehatxatuen zerrendetan sartuta dago eta, ondorioz, gure ondare naturalaren kontserbazio eta kudeaketaren ikuspegitik espezie interesgarria da. Euskal Autonomia Erkidegoan (EAE) interes bereziko gisa sailkatuta dago; gainera, Espainiako Espezie Mehatxatuen Katalogoan sartuta dago, baita Bernako Hitzarmeneko II. eranskinean ere. Artikulu honen helburua 
da EAE guztirako errekako ur-zozoen errolda metodo bat proposatzea, okupazio ereduetan oinarrituta. Horrela, lurralde mailako metodo erabilgarri bat eskainiko da populazioaren banaketan (eta, zeharka, ugaritasunean) aldaketak epe luzera zehaztu ahal izateko. Aurreko ikerketa batean Gipuzkoarako laginketa-puntuen kopuru hoberena aurkitzeko kalkulatutako $\psi$ (okupazio probabilitatea) eta p (espeziea detektatzeko probabilitatea presentzia badago) kontuan izanik, 199 laginketa-puntuko sare bat lortu da EAE guztirako (Arabako eremu mediterraneoa kanpo utzita); ikuspegi espazialetik orekatua (probintzia bakoitzeko ibaien luzeraren araberakoa). Metodo hori ezartzeak aukera emango luke ikergai den espeziearen zentsua egiteko, epe luzerako, modu estandarizatuan, eta haren kontserbazio egoeraz balore markatzaile bat lortzeko. Horrez gain, zentsua egiteko metodoa erreketako beste espezie batzuekin ere erabilgarria izango litzateke, hala nola Alcedo atthis L., 1758 martin arrantzalea eta Motacilla cinerea L., 1758.

Gako hitzak: Erreka hegaztiak, hegazti paseriformeak, segimendu-programak, okupazio modeloak

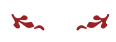

\section{Introducción}

Según la Directiva Aves, los Estados que conforman la UE tienen como obligación el desarrollo de seguimientos para determinar el estado de conservación de poblaciones y especies. En este contexto, son múltiples los programas que, en toda Europa, se desarrollan para determinar tendencias a largo plazo en la distribución y el tamaño de las poblaciones de aves (Peach et al., 1998, Bani et al., 2009, Garrido et al., 2012, Arizaga et al., 2013). La aplicación de metodologías de censo adecuadas es fundamental para una correcta estimación de los parámetros que se desean calcular.

El mirlo acuático Cinclus cinclus L., 1758 es un paseriforme politípico distribuido en casi todo el Paleártico, desde el oeste de Europa (Portugal) hasta Asia Oriental (Cramp, 1988). En general, la especie se asocia a cursos fluviales de agua limpia, poco profunda, rica en macroinvertebrados (Ormerod \& Tyler, 2005). En el sur de Europa, la especie se localiza, mayoritariamente, en ríos de montaña (Ormerod \& Tyler, 2005). En el área cantábrica, no obstante, es común en toda la región (Aierbe et al., 2001, López et al, 2003). En la Comunidad Autónoma Vasca (CAV) es común en las cuencas del área cantábrica y se rarifica en la región mediterránea, reproduciendo de este modo el patrón descrito en España a escala regional (López et al., 2003).

La dependencia del mirlo por los cursos fluviales de agua limpia y en buen estado de conservación ha llevado a su consideración como especie de interés en el ámbito de la bioindicación (Ormerod \& Tyler, 1993, Tyler \& Ormerod, 1994). Por otro lado, está incluida en diversos listados de especies amenazadas y es, en consecuencia, una espe- 
cie que tiene interés desde el punto de vista de la conservación y gestión de nuestro patrimonio natural. En particular, en la CAV está catalogada como de Interés Especial; está incluida, además, en el Catálogo Español de Especies Amenazadas, así como en el Anexo II del Convenio de Berna.

El conocimiento sobre la evolución de la población de esta especie en la CAV es relativamente limitado. Se conoce bien su área de distribución (Álvarez et al., 1985, Aierbe et al., 2001), pero sólo parcialmente su tendencia poblacional (Martínez, 2007). Generalmente, en Europa la especie se ha censado a través de transectos, consistentes en recorrer la orilla de los cauces con el fin de contar el número de aves, lo cual permite estimar densidades (e.g., aves/km) (Peris et al., 1991). A diferencia de otros ríos donde esta metodología es útil (Tyler \& Ormerod, 1994), en el caso de la CAV se da la circunstancia de que, debido a la gran cantidad de vegetación ribereña e infraestructuras (escolleras, edificaciones, etc.), a menudo no es viable hacer transectos desde la orilla. La alternativa, consistente en vadear los cauces, a menudo es dificultosa, ya que con frecuencia se avanza poca distancia por unidad de tiempo (dado que los ríos tienen tramos profundos, presas, etc.). El censo desde kayak, utilizado en otras zonas, no es viable en la mayor parte de los ríos de la CAV dada su morfología. A parte de estas limitaciones descritas anteriormente, la precisión de las tres metodologías puede resultar cuestionable, puesto que durante el tiempo de muestreo las aves se mueven por el río, de tal manera que pueden no ser detectadas, o bien contadas en múltiples ocasiones. Asimismo, la correcta aplicación de este método supone un gran esfuerzo de muestreo difícil de mantener a largo plazo. En términos globales, el uso de transectos lineales no parece un método válido para censar la especie en toda la CAV, lo que hace muy difícil obtener estimas de densidad y, en consecuencia, índices poblacionales basados en esta variable. Alternativamente, lo que se puede hacer es obtener índices o estimaciones de presencia y ausencia. La presencia de mirlos podría determinarse mediante transectos, pero surge de nuevo aquí la problemática asociada a recorrer este tipo de ríos y, así, la dificultad de obtener medidas estandarizadas y representativas. Un método alternativo es la aplicación de modelos binomiales simples (D'Amico \& Hemery, 2003), basados en puntos fijos de muestreo, en los que se estima, solamente, la presencia de la especie.

En términos globales, este método consiste en establecer una red de puntos de muestreo, de tal modo que se estima la probabilidad de que la especie esté presente (probabilidad de ocupación psi, $\psi$ ) y la probabilidad de detectar la especie si está presente (probabilidad de detección p). Dicho de otro modo, este tipo de modelos calcula el porcentaje de puntos de muestreo donde la especie aparece, pero corregido para el parámetro $\mathrm{p}$ (donde $\mathrm{p}<1$ ). Dados $\psi$ y $\mathrm{p}$, así como el valor de varianza deseable (dispersión de los datos que se está dispuesto a asumir), se calcula el número óptimo de puntos de muestreo y el número de visitas por punto que deben realizarse para detectar la población nidificante en la zona de estudio (D’Amico \& Hemery, 2003). 
El objetivo de este artículo es proponer un método de censo de mirlo acuático para toda la CAV, basado en modelos de ocupación. Esto se hace con el fin de dotar al territorio de un método útil para determinar, a largo plazo, cambios en la distribución (e, indirectamente, abundancia) de la población. En particular, el objeto de este artículo es proponer una red de censo de mirlo acuático para la $C A V$, basada en puntos fijos de muestreo.

\section{Área de estudio y métodos}

\section{Consideraciones previas}

Para poner a punto la metodología de muestreo (número mínimo de visitas, tiempo de muestreo en cada visita, etc.) se trabajó con datos previos de diferentes ríos de la provincia de Gipuzkoa. En primer lugar, se llevó a cabo una simulación, mediante el programa SODA (Devictor et al., 2010), con el fin de determinar el número óptimo de puntos de muestreo para Gipuzkoa. Partimos de un escenario en el cual consideramos 60 puntos de muestreo, que era el número de puntos de muestreo que podían ser asumidos en un inicio, y con un máximo de 6 visitas, lo cual resultaría en un esfuerzo total de muestreo de 360 visitas. Se trataría, en todo caso, de un esfuerzo presumiblemente adecuado, de acuerdo a estudios previos llevados a cabo en otras zonas (D’Amico \& Hemery, 2003). Para las simulaciones que se explican a continuación, el valor de referencia, en cuanto a esfuerzo de muestreo, es 360 visitas en todos los casos.

Atendiendo a valores de $\psi$ y p reportados en estudios de referencia sobre el mirlo acuático (D’Amico \& Hemery, 2003), se calcula el número mínimo de visitas que debería llevarse a cabo para mantener una varianza baja a la hora de estimar. De otro modo, la estima de se asociaría a una varianza tan alta que se pondría en compromiso la capacidad de detectar cambios en la tasa de ocupación a lo largo del tiempo. En nuestro estudio se asumió un valor de $\psi=0,6$, que es el que se encontró en una población del Pirineo Central (D’Amico \& Hemery, 2003). Debido a la alta densidad de vegetación en las orillas de los ríos de Gipuzkoa, era posible que el valor de p en Gipuzkoa pudiera ser inferior al registrado en Pirineos ( $p>0,5)$, por lo que se consideraron tres escenarios donde el valor de p varió entre 0,8 (alta detectabilidad), 0,6 (detectabilidad moderada) y 0,4 (detectabilidad baja). Además, asumimos una varianza inicial de $\psi$ de 0,0056 (D'Amico \& Hemery, 2003).

En un primer paso, se determinó el número óptimo de puntos de muestreo y visitas, asumiendo los valores que se han descrito arriba de $\psi, p$, varianza y esfuerzo de muestreo. La simulación para determinar el número óptimo de puntos de muestreo dados un valor de $\psi$ constante $(\psi=0,6)$ y p variable (de 0,8 a 0,4$)$ sugirió 180 puntos de 
muestreo y 2 visitas para un valor de $p=0,8$, ó 72 puntos de muestreo y 5 visitas para un valor de $p=0,4$ (Tabla 1). No obstante, asumir para Gipuzkoa una red de 180, o incluso 72 puntos de muestreo, resultaría poco realista desde un punto de vista logístico, por lo que se optó por mantener los 60 puntos de muestreo.

\begin{tabular}{|cccc|}
\hline Detectabilidad & $\mathbf{N}^{\circ}$ óptimo visitas & $\mathbf{N}^{\circ}$ óptimo lugares & Varianza estimada \\
\hline$p=0,8$ & 2 & 180 & 0,0016 \\
$p=0,6$ & 3 & 120 & 0,0025 \\
$p=0,4$ & 5 & 72 & 0,0044 \\
\hline
\end{tabular}

Tabla 1.-Valores simulados de número óptimo de visitas y lugares de muestreo y varianza, según el valor de detectabilidad (bajo, medio, alto). En todos los casos se parte de un escenario en el cual: $\psi=0,6, p=0,4,0,6 \circ 0,8$; varianza $=0,0056$ y esfuerzo de muestreo es 360 (60 puntos de muestreox6 jornadas de censo por punto). El programa SODA se aplicó considerando convergencia asintótica (varianza = MSE), opción "find design", con 10.000 iteraciones.

Table 1.- Simulated values of the optimal number of visits, sampling points and variance for low, medium and high values of detectability. In all cases, the starting scenario is: $\psi=0.6, p=0.4,0.6$ or 0.8 ; starting variance $=0.0056$, sampling effort $=360$ (60 sampling points $\times 6$ visits). Simulations were carried out using the program SODA, assuming an asymptotic convergence (Variance $=$ MSE), with 10,000 iterations, and using the "find design" option.

Posteriormente, llevamos a cabo otra simulación con el fin de comprobar el efecto del número de visitas en el comportamiento de la modelización, asumiendo: $\psi=0,6$, p variable (de 0,8 a 0,4), esfuerzo de muestreo $=360$, puntos de muestreo $=60$. Observamos cómo a partir de 6 visitas, la varianza simulada y la incertidumbre de la estima de $\psi$ se estabilizan (Fig. 1), por lo que cabe interpretar que con un esfuerzo de 60 puntos de muestreo y 6 visitas se alcanza un equilibrio entre el valor de incertidumbre y la precisión del estimador .

A partir de aquí, la distribución espacial de puntos se estableció al azar aunque espacialmente balanceada, esto es el número de puntos por río dependió de la longitud de su cauce. Esto sirvió para evitar la concentración de muchos puntos en unos pocos ríos y la obtención de un cierto equilibrio territorial en el muestreo. Para ello se empleó el método GRTS (Generalized Random Tessellation Stratified sampling) (Etxaniz, 2005), aplicado mediante el comando "grts" del paquete para R "spsurvey" (Bonney et al., 2009). El muestreo se desarrolló entre los meses de marzo y mayo de 2015 y 2016. Durante estos meses, en 2015 cada uno de los puntos se visitó una vez por quincena (un total de 6 visitas en cada uno de los puntos), durante un periodo de 10 min por visita, preferiblemente durante la primera mitad de la mañana. Posteriormente, en 2016 se repitió el censo, si bien esta vez sólo en 10 puntos donde la especie se había detectado en 2015. Esta vez, los puntos fueron visitados durante un periodo de 20 min, con el fin de detectar si un incremento en el tiempo de muestreo permitiría 

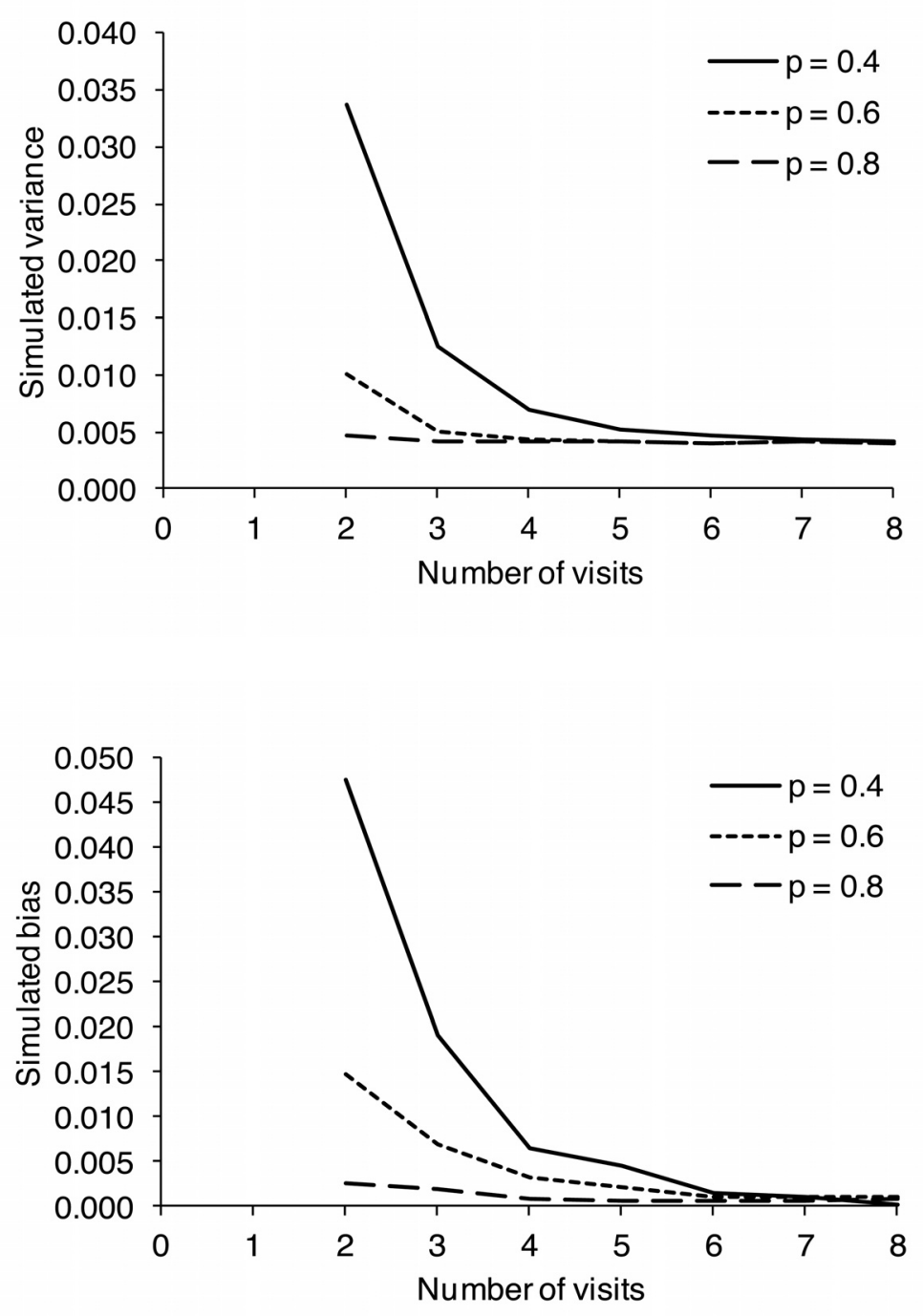

Fig. 1.- Efecto en la varianza simulada (arriba) y la precisión de en la estima de $\psi$ (abajo) para un número variable de visitas y valores de detectabilidad, asumiendo: $\psi=0,6, p=0,4,0,6$ o 0,8; varianza (inicial) $=0,0056$ y esfuerzo de muestreo $=360$ (60 puntos de muestreo 6 visitas). El programa SODA se aplicó considerando convergencia asintótica (varianza = MSE), opción "find design", con 10.000 iteraciones.

Fig. 1.- Effect of the number of visits on simulated variance (above) and the bias of the occupancy probability estimator (below) as obtained in a scenario assuming: $\psi=0.6$, sampling effort $=360, p$ equal to $0.8,0.6$ or 0.4 . Simulation carried out using the SODA software assuming an asymptotic convergence (variance $=$ MSE), with 10000 iterations. 
reducir el número de puntos de muestreo al incrementarse la detectabilidad (p), o bien reducir el número de visitas. Sólo se consideraron aves adultas pues el trabajo se centraba en la población nidificante y no interesaba contar posibles aves jóvenes en proceso de dispersión. En esas fechas, el plumaje de adultos y jóvenes es muy diferente por lo que no resulta difícil su identificación (adultos: aves pardas, más oscuras por arriba que por debajo, con babero blanco; jóvenes: aves grisáceas, con un leve moteado blanco) (Cramp, 1988).

Considerando los valores que se obtuvieron en el muestreo de 2016 para $\psi$ y p ( $\psi=$ 0,$51 ; p=0.52$ ), se comprobó que (1) la potencia estadística llegaba a estabilizarse a partir de 4 visitas, y (2) la varianza y la precisión de la estima de $\psi$ mejoraba (se reduce) al considerar un muestreo de 20 min en lugar de 10 min. En consecuencia, y con el fin de reducir el esfuerzo de muestreo al máximo, asumimos que una red de 60 puntos de muestreo en Gipuzkoa, considerando un muestreo de 20 min por visita y 4 visitas por cada uno de los puntos de muestreo, se ajusta a un diseño que permita detectar cambios en la distribución de la especie de estudio sin perder potencia.

\section{Diseño de la red de censo de mirlo acuático en la CAV}

Para el diseño de una red de puntos de muestreo de la especie de estudio en la CAV primeramente se empleó como capa de origen la capa de masas de agua fluviales de la CAV, publicada en geoeuskadi. Esta capa deja fuera las masas de agua costeras, de transición y embalses que, por otro lado, son hábitats donde el mirlo acuático no cría. Por otro lado, desde esta misma fuente de datos se descargó la capa de regiones biogeográficas de la CAV, que se usó para cortar la capa anterior y seleccionar sólo los ríos de la región atlántica, ya que la especie no está o se rarifica significativamente en la región mediterránea (López et al., 2003).

Hecha esta selección, y una vez comprobados los resultados con 60 puntos de muestreo para Gipuzkoa y determinado el tiempo óptimo de muestreo en cada visita, se empleó el paquete para R "spsurvey" (Bonney et al., 2009) con el fin de establecer una red de puntos de muestreo de mirlo acuático para la región atlántica de la CAV. Para calcular el número de puntos en las otras dos provincias se usó un muestreo balanceado, como se ha indicado arriba para Gipuzkoa, teniendo en cuenta la longitud de los cursos en cada una de las provincias (Gipuzkoa: 673,1 km; Bizkaia: 777,9 km; Álava: 782,6 km).

\section{Resultados}

Asumiendo los resultados del estudio piloto llevado a cabo en Gipuzkoa (para más detalles ver Área de estudio y Métodos), obtenemos para el conjunto de la CAV, exclu- 


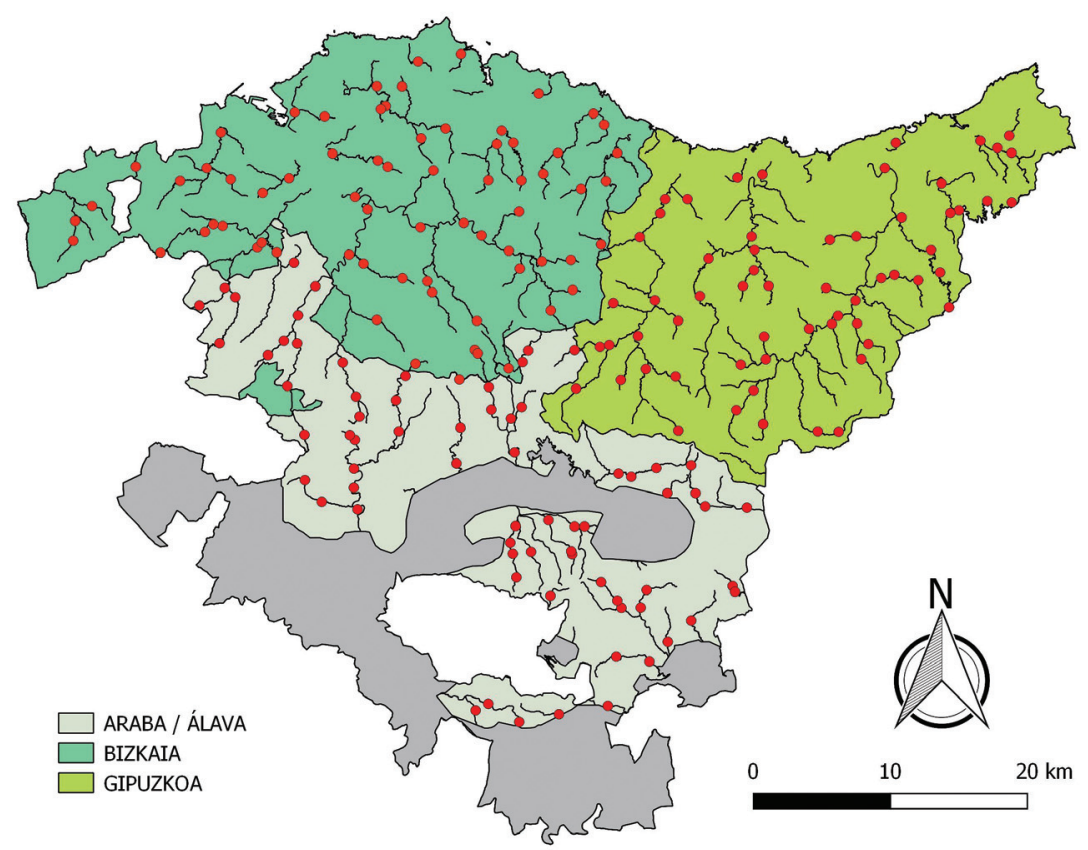

Fig. 2.- Propuesta de puntos de censo de mirlo acuático en la región atlántica (provincias coloreadas; puntos en rojo). En conjunto, se tuvieron en cuenta 199 puntos de muestreo. La zona en gris corresponde a la región mediterránea, excluida del muestreo.

Fig. 2.- Proposal for a network to survey white-throated dippers based on fixed sampling points within the Atlantic region (coloured regions). Overall, we considered 199 sampling points. The grey area corresponds with the Mediterranean region, excluded from this survey protocol.

yendo la región mediterránea, una red de 199 puntos fijos de muestreo de mirlo acuático bien repartida en toda la Comunidad (Fig. 2).

En apartado de Material Suplementario se detalla la relación de puntos, con sus coordenadas.

\section{Discusión}

Hasta la actualidad, la CAV carecía de propuesta alguna orientada a censar la población de mirlo acuático de manera estandarizada en todo el territorio. El método aquí propuesto se basa en censos a llevar a cabo desde puntos fijos y en la orilla. Al evitar entrar en el río y recorrerlo se facilita enormemente la labor de censo y, pensamos, se plantea un protocolo de muestreo cómodo y viable a largo plazo. Esto es fundamental, especialmente si todos o parte de los muestreos se llevaran a cabo mediante voluntariado. 
Teniendo en cuenta la longitud de los ríos que conforman cada provincia (en Álava se ha excluido la región mediterránea, ya que en ésta la especie se rarifica) (López et al., 2003), obtenemos una red de 199 puntos de muestreo. En caso de que un punto fuera de acceso complicado o imposible, cabría elegir otro punto cerca que sustituiría al anterior. Puesto que en cada punto debe invertirse un tiempo de 80 min por año, el esfuerzo de muestreo para toda la CAV asciende a 15.920 min por año (i.e., ca. 265 h). A título comparativo, para un total de 24 cuadrículas en el programa SACRE, de la Sociedad Española de Ornitología, para el censo de aves comunes, el esfuerzo de muestreo global es de 4.800 min (200 min/cuadrícula) (Garmendia et al., 2006). El esfuerzo de muestreo para el mirlo acuático sería, en base a esta referencia, comparativamente alto. No obstante, proponemos que el censo de mirlo acuático no se haga con una periodicidad anual (como ocurre en el SACRE), sino cada 2 (preferiblemente) o 4 años. Además, el censo de mirlo acuático podría utilizarse, también, para otras aves riparias tales como el martín pescador Alcedo atthis L., 1758 y la lavandera cascadeña Motacilla cinérea L., 1758. Otro factor que hay que considerar es el número de participantes. En el estudio llevado a cabo en Gipuzkoa participaron un total de 16 voluntarios. Para 60 puntos de muestreo, 4 visitas y 20 min de muestreo en cada visita, arroja un promedio de $300 \mathrm{~min} /$ voluntario, esto es $5 \mathrm{~h}$ de muestreo/voluntario. Este es un esfuerzo asumible, por lo que proponemos un esfuerzo de 3 a 4 puntos de muestreo/voluntario.

Aunque la división de la red de muestreo en provincias es arbitraria (bastaría con considerar toda la zona atlántica como un solo bloque), consideramos que tal división tiene su punto práctico, ya que la organización de voluntariado y programas de censo en la CAV a menudo se estructura a escala provincial.

Más allá de los objetivos que conciernen a este artículo, el método aquí propuesto podría emplearse a escalas geográficas mayores, e.g. estatal. En general, las aves que se asocian a cursos fluviales (en especial los pequeños paseriformes) no suelen tener una buena representación en los censos de aves comunes y tampoco suelen llevarse a cabo censos orientados a muestrear tales especies (ver la sección de los censos de aves en España en www.seo.org ).

\section{Agradecimientos}

Participaron en la labor de campo (estudios piloto llevados a cabo en Gipuzkoa): A. Arnaiz, A. Díez, A. Erkiaga, A. Galdos, A. Urruzola, E. Iriarte, F. Ansorregi, H. Beñaran, J. Amundarain, J. Etxezarreta, J. I. Jauregi, J. Ugarte, L. Arbeola, N. Pagaldai, T. Aierbe, $X$. Saralegi. Este estudio ha sido parcialmente financiado por la Diputación de Gipuzkoa y el Gobierno Vasco. D. Fernández-Bellón e I. de la Hera aportaron valiosos comentarios que ayudaron a mejorar una primera versión del trabajo. 


\section{Bibliografía}

Aierbe, T., Olano, M., Vázquez, J. 2001. Atlas de las aves nidificantes de Gipuzkoa. Munibe, Supl. 52. Sociedad de Ciencias Aranzadi. Donostia.

Álvarez, J., Bea, A., Faus, J. M., Castién, E., Mendiola, I. 1985. Atlas de los vertebrados continentales de Álava, Vizcaya y Guipúzcoa (excepto Chiroptera). Gobierno Vasco, Vitoria.

Arizaga, J., Crespo, A., Iraeta, A. 2013. Noticias EMAN, 1. Sociedad de Ciencias Aranzadi. Donostia.

Arizaga, J., Resano-Mayor, J., Villanúa, D., Alonso, D., Barbarin, J. M., Herrero, A., Lekuona, J. M., Rodríguez, R. 2018. Importance of artificial stopover sites through avian migration flyways: a landfill-based assessment with the White Stork Ciconia ciconia. Ibis 160: 542-553.

Bani, L., Massimino, D., Orioli, V., Bottoni, L., Massa, R. 2009. Assessment of population trends of common breeding birds in Lombardy, Northern Italy, 1992-2007. Ethology, Ecol. \& Evol. 21: 27-44.

Bonney, R., Cooper, C.B., Dickinson, J., Kelling, S., Phillips, T., Rosenberg, K.V., Shirk, J. 2009. Citizen Science: A Developing Tool for Expanding Science Knowledge and Scientific Literacy. Bioscience 59: 977-984.

Cramp, S. 1988. Handbook of the Birds of Europe, the Middle East and North Africa. Vol. 5. Oxford University Press. Oxford.

D'Amico, F., Hemery, G. 2003. Calculating census efficiency for river birds: a case study with the White-throated Dipper Cinclus cinclus in the Pyrenees. Ibis 145: 83-86.

Devictor, V., Whittaker, R.J., Beltrame, C. 2010. Beyond scarcity: citizen science programmes as useful tools for conservation biogeography. Divers. Distrib. 16: 354-362.

Etxaniz, M. 2005. Seguimiento de la comunidad de aves de Plaiaundi y Jaitzubia (Txingudi). Informe anual 2005. Gobierno Vasco. Inédito.

Garmendia, A., Cárcamo, S., Schwendtner, O. 2006. Forest nanagement considerations for conservation of Black Woodpecker Dryocopus martius and White-backed Woodpecker Dendrocopos leucotos populations in Quinto Real (Spanish Western Pyrenees). Biodivers. Conserv. 15: 1399-1415.

Garrido, J.R., Molina, B., Del Moral, J. C. 2012. Las garzas en España, población reproductora e invernante en 2010-2011 y método de censo. SEO/BirdLife. Madrid.

López, V., Vázquez, X., Gómez-Serrano, M.A. 2003. Mirlo Acuático Cinclus cinclus. In: Atlas de las aves reproductoras de España. R. Martí, J.C. del Moral (eds.): 406-407. SEO/BirdLifeMMA. Madrid.

Martínez, J.M. 2007. Estado de la biodiversidad fluvial de Bizkaia. Evolución de sus poblaciones de Mirlo Acuático. S. O. Lanius. Bilbao.

Ormerod, S.J., Tyler, S.J. 1993. Birds as indicators of changes in water quality. In: Birds as monitors of environmental change. R.W. Furness, J.J.P. Greenwood (eds.): 179-216. Chapman \& Hall. London. 
Ormerod, S.J., Tyler, S.J. 2005. Family Cinclidae (Dippers). In: Handbook of the Birds of the World. Vol 10. J. del Hoyo, A. Elliot, D.A. Christie (eds.): 332-355. Lynx Edicions. Barcelona.

Peach, W.J., Baillie, S.R., Balmer, D.E. 1998. Long-term changes in the abundance of passerines in Britain and Ireland as measured by constant effort mist-netting. Bird Study 45: 257275.

Peris, S.J., González-Sánchez, N., Carnero, J.I., Velasco, J.C., Masa, A.I. 1991. Algunos factores que inciden en la densidad y poblacióon del mirlo acuático (Cinclus cinclus) en el centro-occidente de la Península Ibérica. Ardeola 38: 11-20.

Tyler, S. J., Ormerod, S. J. 1994. The Dippers. T \& A. D. Poyser. London.

Fecha de recepción/ Date of reception: 25/03/2018

Fecha de aceptación / Date of acceptance: 31/05/2019

Editor Asociado / Associate editor: Iván de la Hera 